\title{
Das permanente Exil. Zu Karl Wolfskehls antimoderner Lyrik
}

\section{Drei Symptome der Emigrationspsychose}

Anfang 1998 erschien der Roman Live Bodies des neuseeländischen Schriftstellers Maurice Gee, der den 'Montana New Zealand Book Award' für 1998 erhielt. Das Buch erzählt eine auf wahren Gegebenheiten basierende Geschichte eines gebürtigen Österreichers und Juden Josef Mandl (wie er im Buch heißt), der in den dreißiger Jahren aus politischen Gründen nach Neuseeland flüchtete. Hieraus eine kurze Passage:

Then [...] I began rushing about like a tourist. I went to Rotorua on the bus and saw the geysers and the boiling mud and Maori children diving for pennies under a bridge. Why am I not where I belong?, I thought. I went to Taupo, the rapids and the falls and more steam hissing and mud plopping, and saw a mountain smoking far away, and thought, Interesting, interesting, but Vienna is where I should be. Back in Auckland, I needed to hear my own language. I needed, for an hour or two, not to translate but just to let my ear hear and my tongue speak, and meet the lost part of myself again. So I went along to the Deutscher Verein, the Ger man club.1

Aus Josef Mandls Erinnerungen spricht nicht nur Heimweh, sondern vor allem die existentielle Angst eines Entwurzelten, seine den kulturellen und gesellschaftspolitischen Traditionen in Europa verwachsene Identität zu verlieren. Hier, "auf Erdballs letztem Inselriff” (Wolfskehl), wählte der Exulant Mandl Selbstbegegnung als Therapie, die das Schwinden des Ichs - samt seiner Muttersprache und seinem Verhältnis zum Vaterland - aufhalten sollte. Der Roman Live Bodies kennt zwar ein Happy end, denn der Status des Exilanten ging nach Kriegsende in den des Immigranten über, aber unversehrt war der während des Krieges als "enemy alien" auf Somes Island internierte Mandl keineswegs, denn das Exil drückte schwer auf die Psyche.

Ein weiteres Zitat: "Selbst mir fällt es schwer, den Kopf hoch zu behalten. Ein großer Teil meines wirklichen Daseins vollzieht sich

1 Gee, Maurice, Live Bodies, Auckland 1998, S. 71. Hervorhebung von mir. 
in und aus der Ferne."2 Hier spricht Karl Wolfskehl. In dem zitierten Brief vom 19. November 1941 kommen - nicht zum ersten und auch nicht zum letzten Mal - die Schwierigkeiten vom Leben im Hier und Jetzt bzw. kommt die Angst vor dem Identitätsverlust zum Ausdruck. Thematisiert wird die sogenannte Emigrationspsychose. Die meisten der 1959 von Margot Ruben herausgegebenen zweihundertfünfundvierzig Briefe, die Karl Wolfskehl vom neuseeländischen Exil aus in alle Welt verschickte, sind $\mathrm{m}$. E. äußerst aufschlußreich für Psychologen und Psychoanalytiker. In ihnen widmet sich Wolfskehl einer Vergangenheit und Welt, die für ihn noch in Ordnung war. Der Briefwechsel dient der Abwehr der Präsenz in einer neuen Welt, steht somit im Zeichen der Regression. Die Rückbewegungen können aus drei herausragenden Symptomen, die sich meist gleichzeitig manifestieren, abgeleitet werden.

\section{Erstes Symptom: Die Kreation eines alter egos}

Insbesondere in der in der zweiten Hälfte der vierziger Jahre initiierten Korrespondenz redet Karl Wolfskehl sich selbst immer häufiger in der dritten Person singular an, vor allem dann, wenn der Briefschreiber sich an den Dichter in ihm wendet. Besonders illustrativ ist der Brief an einen gewissen Herrn " $X$ " vom 4. April 1947, in dem Karl Wolfskehl eine nach seinem Verständnis ungemäße, aus dem Brief jedoch nicht zu erschließende Kritik am Werk Karl Wolfskehls zurückweist: "Heute muß ich Ihnen sehr ernstlich vorhalten, dass ich mit Ihren Ansichten über Art und Richtung des dichterischen Werks von Karl W'olifskehil nicht divertiert zu werden wünsche." 3 Es sind die existentiellen Nöte, die Zweifel und die Ängste angesichts des Verlorenen, welche eine ständige Konfrontation mit der eigenen Vergangenheit in der Form einer rückwärtsgewandten Selbstdarstellung und -bestätigung evozieren. Um die vergangene Ganzheit nicht völlig der Zerstückelung und Verfremdung anheim zu geben, muß Einheit simuliert, müssen Dialogpartner auch von seinem eigenen Subjekt abgespalten werden, um wenigstens teilweise die Verbindung mit dem Vergangenen herstellen zu können.

2 Ruben, Margot (Hg.), Karl Wolfskehl. Zehn Jahre Exil. Briefe aus Neuseeland 1938-1948, Heidelberg und Darmstadt 1959, S. 100 (Fortan: BaN I).

3. BaN I, S. 327. 
Ein Blick in das Namenregister der Ausgabe Zehn Jahre Exil. Briefe aus Neuseeland gibt einen weiteren Tiefblick in die Psyche des exilierten Dichters: Der Eintrag "George, Stefan [Meister]" ist der längstgeratene im Index. Vom Anfang bis zum Ende des Briefwechsels taucht immer wieder die Person und das Werk Stefan Georges auf, der von früh an von Wolfskehl zur mythischen Gestalt verklärt wurde, weil dieser "alles vorgewusst, vorgesprochen" hat.4 Noch im neuseeländischen Winter 1947 zum Beispiel beschwor der Briefschreiber den ewigen geistigen Bannkreis, der dem 'Meister' umgab:

Das Werk des Meisters trägt man in sich, oder besitzt es überhaupt nicht, auch wenn es in all seinen Editionen um uns stünde. Was ich von Zufälligem brauche, das doch Keiner von uns entbehren möchte, ich vielleicht am wenigsten von Euch allen, ersetzen eben die Zurufe von fernher [...].5

Die geistige Geborgenheit, die George verspricht und die kulturelle Sprengkraft, die sich in der Ferne immer aufs Neue entlädt, macht das Vorbild allgegenwärtig. Für den verlorenen Sohn wird George zur Vaterfigur.

\section{Drittes Symptom: Die endgültige Selbstaufgabe}

Insbesondere in den letzten Lebensjahren setzt Wolfskehl sein Einzelschicksal mit dem Los der Juden, der jüdischen Diaspora und generell mit dem Verlust der Heimat gleich. Über das Verfahren der Übertragung des Schmerzes hat Paul Hoffmann ausführlich in seinem Aufsatz "- jüdisch, römisch, deutsch zugleich" geschrieben. Darin erkennt Hoffmann, daß Wolfskehl vor allem im Spätwerk als Repräsentant jener geistigen und für seine Arbeit konstitutiven Welten auftritt.6 Die Projektion ging viel weiter: Die Kom-

4 BaN I; S. 65.

5 BaN I, S. 340. Wolfskehl kann keineswegs als der "polare Gegenpart" Stefan Georges bezeichnet werden, wie Fritz Usinger das 1959 in seinem Nachwort zu den Neuseeland-Briefen tat. Siehe BaN I, S. 383.

6 Vgl. Hoffmann, Paul, “"- jüdisch, römisch, deutsch zugleich.' Karl Wolfskehl.". In: G. Grimm und H. P. Bayersdörfer (Hg.), Im Zeichen Hiobs. Jüdische Schriftsteller und deutsche Literatur im 20. Jahrhundert, Königstein/Ts 1986, S. 115. 
bination der religiösen Rhetorik mit der neuen Erfahrung mit dem Bösen mündete in eine Inkarnation Hiobs und in eine Gleichsetzung des Hiob- und Wolfskehl-Loses. Er sah sich dazu bestimmt, "[...] das lebendige, ja das schaffende Symbol dieses Schicksals darzustellen."7 Die Selbstinszenierung führte jedoch zur Selbstaufgabe, die eine Analyse der horrenden politischen Sachzwänge, deren Resultat seine Verbannung war, nicht zuließ.

Die drei Symptome deuten auf einen Verzicht auf die Autonomie des Ichs. Es steht im Dienst des Anderen, wodurch sich der Repräsentant der Vergangenheit zwangsläufig von jedweder Modernität und Aktualität, d. h. von einem zugespitzten Ausdruck eines Zeitbewußtseins, das die Gegenwart als krisenhaft erfährt bzw. diese wenigstens problematisiert, fernhält. Wolfskehl verschließt sich der "Konkretisierung des gesellschaftlichen Sündenfalls der Deutschen".8 - Briefe aus dem Exil, so schrieb Gert Mattenklott 1993, dienten vielmehr der wiederholten "innere[n] Begegnung" und "symbolische[n] Gemeinde und Gemeinschaft": "Je prekärer es mit den weltlichen Ordnungen bestellt war, während die geistlichen ungewiß verdämmerten, desto höheren Wert mußten im Niemandsland der metaphysischen und weltlichen Anarchie die freiwillig eingegangenen Verbindlichkeiten erhalten: als Formen moralischer Selbstbegegnung."9 Nachdem Mattenklott näher auf die Bedeutung des Mediums Brief als genuine Form jüdischer Selbstfindung und generell auf die bürgerliche, europäische Tradition des Briefwechsels eingeht, stellt er die These auf, daß eine Antwort auf gerade jene Briefe, die Juden im Exil schrieben, nicht unbedingt nötig war, sondern die Enthüllung der privaten Gedanken und Gefühle die Gegenwärtigkeit des Dialogpartners ersetzte. Die Korrespondenz Wolfskehls liest sich als langer Monolog und spricht vor allem von der Angst vor dem Verlust des Obdachs. Zwangsläufig, so Mattenklott, tendiert der Inhalt dieser Briefe $\mathrm{zu}$ einer "nur noch in sich selbst kreisenden Welt".10

Die Tragik dieser Gegenwartsfremde kann m. E. nicht nur mit einer

7 BaN I, S. 285.

8 Klein, Alfred, “'Die Stimme spricht'. Anmerkungen zu einem Gedichtband von Karl Wolfskehl (1869-1948)." In: A. Klein, K. Pezold und W. Schubert (Hg.), Literarische Streifzüge. Für Hans Mayer von Schülern der Leipziger Zeit. Leipzig 1996, S. 200.

9 Mattenklott, Gert, "Nelly Sachs, Arnold Zweig und Karl Wolfskehl - Briefe aus dem Exil." In: I. Shedletzky und H. O. Horch (Hg.), Deutsch-jüdische Exilund Emigrationsliteratur im 20. Jahrhundert, Tübingen 1993, S. 140.

10 Ebd., S. 145. 
(selbstredend professionelleren) Psychographie erfaßt bzw. mit dem traurigen zehnjährigen Status des isolierten Exul Poeta oder mit dessen Erblindung erklärt werden, sondern sollte ebenfalls mit der spezifischen Ästhetik, die Karl Wolfskehl von Anfang seiner literarischen Laufbahn an verfolgte, in Beziehung gesetzt werden. Insbesondere aus der Lektüre der frühen Lyrik geht eine bewußte Zeitablehnung hervor, die sich in dem lyrischen Spätwerk radikalisierte. Auf diese Weise entstand eine Poesie, die sich in einem selbstauferlegten Exil bzw. einem Abwenden vom Mainstream der modernen Zivilisation in Deutschlands Zweitem Kaiserreich und später - erster sozialdemokratischer Republik fortbewegte, um eine untergehende geistige Kultur konservieren zu können. Diese "hermetische Kraft" fiel Walter Benjamin 1929 in seiner Erinnerung an den damals sechzigjährigen Dichter auf, als dieser von Wolfskehls Leben und Werk als "weltgeschichtliche[m] Refugium" sprach.11 Angeregt vom für mich provozierenden Adjektiv "elastisch", das Guy Stern 1998 in seinem Buch Literarische Kultur im Exil in die Debatte der gegenwärtigen Exilforschung eingeführt hat,12 möchte ich zunächst die Kategorie des Exils erweitern und versuchen, diese auf Stil- und Strukturmerkmale lyrischer Texte anzuwenden. Dabei werde ich mich vor allem auf den Umgang mit dem lyrischen Subjekt und der Kategorie der Wiederholung in Wolfskehls Gedichten beschäftigen. Auffallend sind zum Beispiel die dem Ich unterliegenden Formzwänge, die ein Sprechen über den Rand des Textes hinaus unterbindet; ferner deutet die Wiederholung von Vorgesprochenem auf eine weitere Ich-Restriktion. Der Vergleich einiger Gedichte aus der Zeit vor und nach dem Stichjahr 1933 sollte die Kontinuität dieser Selbstentsagung aufzeigen, welche in eine - wie ich meine - "Exul Poetica" mündete. Im letzten Teil werde ich Wolfskehls Schreibweise und seine eigenwillige Poetik im Rahmen des Diskurses, der den sogenannten deutschen Antimodernismus prägte, besprechen. Seine Lyrik steht im Zeichen eines am Anfang des 20. Jahrhunderts im George-Kreis beschworenen ästhetischen Fundamentalismus, der sich in der Zeit seiner Verbannung in Neuseeland fortsetzte.

11 Benjamin, Walter, "Karl Wolfskehl zum sechzigsten Geburtstag. Eine Erinnerung." In: Ders., Gesammelte Schriften IV. 1. Hg. von Tillman Rexroth. Frankfurt a. M. 1972 , S. 368.

$12 \mathrm{Vgl}$. Stern, Guy, Literarische Kultur im Exil. Gesammelte Beiträge zur Exilforschung. Literature and Culture in Exile. Collected Essays on the GermanSpeaking Emigration after 1933. (1987-1997), Dresden 1998, S. 7. 


\section{Exul Poetica}

Das Motto des Gedichts "Nach fünfzig Jahren" aus dem in Neuseeland konzipierten Großzyklus Die drei Welten und das Lebenslied läßt eine zeitliche Zweiteilung vermuten. Das Motto lautet: "Die beiden ersten Strophen beginnen ein Gedicht des Neunzehnjährigen." 13 Im auf diese Weise markierten ersten Teil heißt es:

Ich war ein fahrend heimatloser Schweifer, Und achtlos ging am Schönen ich vorbei, Doch da ergriff mit wildem Liebeseifer Das junge Herz mir eine mächtige Fei.

Urplötzlich sah ich bunte Blumen spriessen Und eine Heimat, eine Ruhestatt Umgab mich. In entzückendem Geniessen Trank ich am Kelch der Liebeslust mich satt.

Aus zeitlicher Distanz werden diese als junger Mann geschriebenen Verse von Wolfskehl selbst als ungelenkig und unreif eingeschätzt. Später im Text wird die mächtige Zauberfee von einer noch mächtigeren, jetzt männlichen, Gestalt ersetzt; seine Hand ergriff und führte ihn. In der letzten Strophe des Gedichts wird die für Wolfskehls Lyrik typische Kreisbewegung vollendet, wobei das Hier und Jetzt in die Vergangenheit eingegliedert wird. 'Nach fünfzig Jahren' ist ein Musterbeispiel der Verschmelzung von Vergangenheit und Gegenwart:

Heut, heimlos zwar, greis, wider Recht gebannt, Schatzhalter doch den unser Meister braucht Heb ich den Kelch, der Flamme ganz Trabant, Bis Schall und Schau in bracher Nacht verraucht.

Das Gedicht verknüpft nicht bloß zwei, sondern insgesamt drei Zeitebenen. Betrachten wir die einzelnen Sequenzen genauer, fällt nicht nur die Kreismotivik, sondern (damit zusammenhängend) eine zunehmende Beeinträchtigung des Wirkungsraums des lyrischen Ichs in den drei hier vorgestellten Schaffensphasen des Lyri-

13 Wolfskehl, Karl, Gesammelte Werke. Erster Band. Dichtungen, Dramatische Dichtungen. Hg. von Margot Ruben und Claus Victor Bock, Hamburg 1960, S. 227 (Fortan: GW I). 


\section{Erste Sequenz: 1888/89}

Die ersten beiden Strophen thematisieren die Anfangsphase, "ein Vierjahr vor Erweckung", d. h. vier Jahre vor der für Wolfskehl folgenschweren Begegnung mit Stefan George, unter dessen Maß der junge Poet sich stellen wollte, wie auch aus den Erinnerungen aus dem Jahre 1928 hervorgeht: "Ich wußte von dem Augenblick [der ersten Begegnung; gjb] an, wohin ich gehöre, was in mir Norm sei [...] Georges geformtes Wort [...]."14 Das Geformte ließ die romantischen Züge (das Schweigen, Genießen, die bunten Blumen und das Entzücken) und die auffälligen Plötzlichkeitsmetaphern (Bohrer), wie sie im ersten oben zitierten Teil des Gedichts vorkommen, nicht mehr zu. Die Absage hatte zur Folge, daß die Jugendgedichte nicht in die Gesammelte[n] Dichtungen von 1903 eingegangen bzw. nicht in den Blätter[n] für die Kunst erschienen sind.

\section{Zweite Sequenz: 1892/93-1933}

Die persönliche Begegnung mit Stefan George in München im August 1893 war mehr als eine Zäsur: sie initiierte eine Neugeburt, die "Erweckung / Durch seinen Herrn". In dieser Georgeschen Phase dominiert der Formzwang, wie in den Gedichten aus den Bänden Gesammelte Dichtungen (1903), Der Umkreis (1927) und Die Stimme spricht (1934/36) hervorgeht. Im Lyrikband Die Stimme spricht, dessen Gedichte eine Distanzierung vom 'Meister' vermuten lassen - u. a. durch das Ablegen der Kleinschreibung, vor allem aber durch die Einführung des Wolfskehlschen Themas der Suche nach dem Göttlichen -, tritt George aber nach wie vor als Korrektor auf. Dieser zeigt dem Gefolgsmann Wege, die ihm sonst verschlossen gewesen wären. In Konkurrenz zu Gott begleitet der 'Meister' (in den Worten Stefan Zweigs: der "ästhetische Papst") das weitere Schaffen, wie aus der vierten Strophe hervorgeht:

Wie war ich seit der Jahre halbes Hundert

Andächtig nach Allgottes goldner Welt,

Vorm Meister still, verrufen und bewundert,

Trunkenstem Augenblicke beigesellt.

14 Zitiert in: Schlösser, Manfred, Karl Wolfskehl 1869-1969. Leben und Werk in Dokumenten, Darmstadt 1969, S. 77. 
Noch im Oktober 1933 schreibt der bereits ins Exil Aufgebrochne im dritten Teil des Gedichts "Aufbruch, Aufbruch": "Der Worte Wort, vom Meister stammend, / Der Liebe Du aus junger Zeit". 15 George, der im Dezember 1933 starb, befindet sich im geistigen Gepäck des Exilanten.

\section{Dritte Sequenz: 1938/39}

Obgleich nicht kritiklos war Wolfskehl Stefan George auch über die vierzig Jahre hinweg verbunden, wie zum Beispiel aus dem Gedicht An die Deutschen hervorgeht. Im zweiten Teil, "Der Abgesang", heißt es: "Weit aus heilig weissem Feuer / Reckt die Hand und heischt der Meister: / Überdauere! Bleib am Steuer!"16 Sogar noch aus dem Jenseits werden die Koordinaten für den Kurs, der auf die Vollendung der Georgeschen Ästhetik samt seines "neuen reiches" zusteuert, durchgegeben. Mit diesem Glaubensbekenntnis spielte er die "wahre Heimat" gegen die auferzwungene "Wahlheimat" aus, denn - so im letzten Zyklus von "Mittelmeer oder Die fünf Fenster" - das "Meisterwort" bringe dem Exul Gewähr.17 Bemerkenswert in dem Gedicht "Nach fünfzig Jahren" ist, daß es nicht zur Absage an die literarischen 'Jugendsünden' kommt und Wolfskehl sich diesen verbunden fühlt. Bezeichnenderweise wiederholt er das Wort "Kelch" aus den ersten beiden Strophen des Neunzehnjährigen in der letzten Strophe, genauer: im neunzehnten Vers des Gedichts. So schließt sich der Kreis, wobei die ersten beiden Zeitebenen nicht korrigiert, sondern integral in die spätere Schaffensphase integriert werden. Das heißt: In den späteren Schaffensphasen ändert sich die Tonart der Lyrik Wolfskehls nicht wesentlich; produktionsästhetisch gesehen, wirkt die Spätlyrik unausgereift, denn sie unterliegt dem Diktat der Wiederholung des früheren Schaffens. Konträr zu den Würdigungen von zum Beispiel Rudolf Pannwitz und Paul Hoffmann, die die im letzten Drittel von Wolfskehls Leben geschriebene Lyrik als Vollendung seines Werks eingestuft haben,18 schließe ich mich der Argumentation von Gunter Grimm an.19 Er analysiert die Exillyrik mit Hilfe von Kate-

15 GW I S. 167.

16 GW I, S. 219.

17 Vgl. GW I, S. 191.

18 Vgl. u. a. Pannwitz, Rudolf, "Der Dichter Karl Wolfskehl." In: M. Schlösser, Wolfskehl, S. 9-21 und Paul Hoffmann, "- jüdisch, römisch, deutsch zugleich." a. a. $\mathbf{O}$. 
gorien der Epigonalität, die bereits Zeitgenossen von Wolfskehl kritisiert haben. Damals, nach der Veröffentlichung von Der Umkreis (1927) zum Beispiel, wurde auf die "Leere" und die "papiere$\mathrm{n}[\mathrm{e}]$ " Wesenslosigkeit der Verse hingewiesen. In den Verrissen wurde Wolfskehls Gelehrtenpoesie in Beziehung zu Georges Menschenleere in seinem künstlichen Paradies gesetzt.20 - Wie hieß es noch einmal im 1892 erschienenen Band Algabal: "Mein garten bedarf nicht luft und nicht wärme". - Die einzigen menschenähnlichen Gestalten in Der Umkreis zum Beispiel sind Gespenster und Gerippe, die neben dem Göttlichen und Mythischen einem anderen Kult dienen, dem Kult der Kälte.

Einerseits scheint Wolfskehl hier zivilisationskritische Gedanken von u. a. Oswald Spengler aufzugreifen und kritisiert er die moderne Zeit am Anfang des zwanzigsten Jahrhunderts mit Bildern, die an die Eiszeit erinnern. Die insbesondere in der Literatur der zwanziger Jahre frequent eingesetzten "Kältemetaphern" (Lethen) dienten der Abrechnung mit der maroden, wenn nicht sogar der zum Tode aufgeschriebenen Zivilisation, so etwa im Gedicht "Menschendämmerung", dessen zweite Strophe wie folgt lautet: "Die lezten leben im kalten / Wollen warm sich halten / Bein reibt bein".21

Andererseits werden die auffallenden klimatischen Metaphern in Wolfskehls vergeblichem Ringen um die Aufnahme in die Wärmezonen des Allmächtigen eingesetzt, was mit der Verbannung des lyrischen Ichs an die kalte Peripherie einhergeht. Als Gegenstrategie dieser Verbannung gilt das Ausprobieren unterschiedlicher Tarnungen des Ichs, um wenigstens auf Umwegen die Nähe zum Göttlichen zu suchen. Aber das Sprechen in fremden Zungen, im Maskenzug sozusagen, versagt, so daß es nicht zur Symbiose mit dem mächtigen 'Anderen' kommt. Die Masken, die Wolfskehl favorisiert, sind übrigens oft selbst der Verbannung verschrieben: Es sind die von u. a. Ovid, Orpheus, Dionysos, Odysseus und

19 Vgl. Grimm, Gunter, Karl Wolfskehl. Die Hiob-Dichtung, Bonn 1972, S. 300: "Eine Neuschöpfung von Form und Sprache ist Wolfskehl zweifellos nicht gelungen. Doch wird dies auch schwerlich seine Intention gewesen sein. Vielmehr handelt es sich beim Spätwerk um eine Weiterentwicklung des eigenen immer auf George basierenden Frühwerks [...]."

20 Vgl. die Kritik von Heinz Lützeler. Zitiert in M. Schlösser, Karl Wolfskehl 1869-1969, a. a. O., S. 31. Vgl. auch Biese, Alfred, Deutsche Literaturgeschichte. Dritter Band: Von Hebbel bis zur Gegenwart, München 1921, S. 709.

$21 \mathrm{GW}$ I, S. 102. Zur Theorie der "Kältemetaphern", siehe u. a. Lethen, Helmut, Verhaltenslehren der Kälte. Lebensversuche zwischen den Kriegen, Frankfurt a. M. 1994. 
natürlich Hiob. Sie sind Spiegelungen, Schemen des Ichs. In den im Exil geschriebenen Hiob-Zyklen ist die Maske Hiobs sogar vierfach gefächert. Gerade hier ist die Selbstaufgabe am größten, wenn die Inkarnation in vier unterschiedlichen Gestalten auftritt. In diesem Spiegelkabinett läßt sich Karl Wolfskehl in mehrfacher Form spiegeln bzw. fragmentieren, wo sich doch Hiob bereits selbst im (eigentlich unsichtbaren) Bild Gottes gespiegelt hatte und das lyrische Ich sich (und nach den Exil-Briefen zu urteilen, Wolfskehl selbst) vier Mal im Schmerzensmann spiegeln läßt, und zwar in Hiob Israel, Simson, Nabi und Machiach. Am Schluß des vierten Spiegels ist das Ich völlig geschrumpft, wenn es nur noch heißt: "WER-ER-DU".22 Im gesamten letzten Zyklus bzw. Spiegel versiegt der Sprachfluß, weicht der Pathos der Wortkargheit, was m. E. nicht - wie Paul Hoffmann es versucht hat - mit den sprachlichen Reduktionen in Paul Celans Gedichten zu vergleichen ist, sei es nur deshalb nicht, weil um den Hiob-Zyklus wortgewaltige Gedichtfolgen, mitreißende Hymnen und pathetisch anmutende Elegien kreisen, die die scheinbaren stilistischen Innovationen neutralisieren.

Die Ich-Restriktion, verbunden mit der Arbeit am Vergangenen wird durch eine oft trümmerhafte Reimordnung und einen oft peinlich anmutenden Reimzwang vermittelt.23 Drei Beispiele dieses Wolfskehl-Sounds aus unterschiedlichen Schaffensphasen sollen die Kontinuität dieser stilistischen Eigenart illustrieren: "Am saume des lebens muss ich liegen / Verdorrt und lästernd muss ich liegen" (aus: Gesammelte Dichtungen); "Bist du noch wirrst du noch fährst noch im sturm, sturm? / Bist du noch irrst du noch bohrst

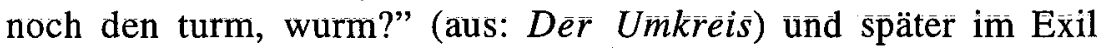
heißt es u. a. "Spürt ihn spürt ihn spürt ihn nicht" oder "Das Wort hat seine Zeit gehabt, / Hat alle seine Zeit gehabt" (aus: "Die drei Welten und das Lebenslied").24 Der Aspekt des Wiederholens, als Rhetorik und Vortragsform jüdischer Gebetsbücher 25 verständlich, wirkt im Gedicht gekünstelt. - Die Form macht die Lektüre anstrengend, da gebremst; sie stagniert letztendlich durch die Anhäufung der Selbstbezüge. Noch anstrengender wirkt sie, wenn die zahlreichen mit der Wiederholung korrespondierenden Kreisbewegungen

\section{$22 \mathrm{GW}$ I, S. 215.}

23 Vgl. die Kritik von Kaiser, Gerhard: Geschichte der deutschen Lyrik von Goethe bis zur Gegenwart. Ein Grundriß in Interpretationen. Band II: Von Heine bis zur Gegenwart, Frankfurt a. M. 1996, S. 274-276.

24 GW I, S. 33, 73 und 176.

25 Vgl. Pearl, Chaim/Brookes, Reuben S., A Guide to Jewish Knowledge, London 1956. 
und -motive ins Auge springen. Bereits 1962 hat Ernst Simon auf den offensichtlichen Manierismus in Wolfskehls Lyrik hingewiesen. Aktueller ist die Kritik von Wolfgang Braungart, der vor allem auf die liturgische Form der Zeitzyklen und die epigonale Wiederverwendung der Georgeschen Bogenführung und Binnenvokalisation hingewiesen hat. Er hat die Verknüpfung des Glaubensbekenntnisses mit dem Austritt des Ichs aus der modernen Gesellschaft sowohl bei Stefan George als auch Karl Wolfskehl und anderen Georgianern auf textueller Ebene aufgezeigt.26

Nun ist eine intellektuelle Herausforderung des Lesers in meinem Verständnis gerade ein positives Merkmal der Lyrik, - im Falle Wolfskehls jedoch handelt es sich nicht um eine sogenannte "Sprachanstrengung", die auf ein poetisches Können und eine damit verknüpfte Innovation schließen läßt, sondern vielmehr um eine "Mache", die zum barocken Schwulststil neigt und den Text zum musealen Gebilde konstruiert. Das Konservieren der dem Untergang anheimgegebenen Kulturen wird nicht befragt, sondern, im Gegenteil, es soll als Pfeiler im zivilisatorischen Chaos Halt gewähren, somit Obdach simulieren. Durch die Fülle werden die Texte jedoch leer, und das Ich kapselt sich von der Welt über dem Rand des Gedichts ab, wird dadurch schemenhaft. Das lyrische Subjekt in Wolfskehls Lyrik tritt (frei nach Hans Mayer) als intentioneller Außenseiter auf, und weiter: es negiert in dieser konstruierten "inneren Emigration" seine geschichtliche Bedingtheit.27

\section{Schreiben im Zeichen der Antimoderne}

Der am 11. April 1947 an Edith Landmann und Renata von Scheliha verfaßte Brief illustriert, mit welcher Vehemenz Karl Wolfskehl seine Geschichtsauffassung, welche sein Dichterprofil prägte, verteidigte. Darin pochte er auf die

[...] Erkenntnisart, die für den Meister und für uns Alle die einzig der Wirklichkeit nahekommende blieb, und deren zentraler Satz heisst: Jedes Ding, jedes Stück Leben, jedes Gebilde muss aus sich

26 Braungart, Wolfgang, Ästhetischer Katholizismus. Stefan Georges Rituale der Literatur, Tübingen 1997.

27 Mayer, Hans, Außenseiter, Frankfurt a. M. 1975. Vgl. auch den Eintrag "Exil" in: Brockhaus' Konversations-Lexikon 1895, S. 466: "Verbannung. Das Altertum bezeichnete damit bald den freiwilligen Austritt, durch welchen ein Bürger dem Volksunwillen zu entgehen suchte (so zur Zeit der röm. Republik Coriolan, Verres, Cicero), bald den Zwang zur Auswanderung mittels Volksbeschlusses [...]." Hervorhebung von mir. 
selber verstanden, erkannt, gedeutet werden. Nichts darf von seiner Stelle gerückt werden. Analogie, und wie dergleichen Hilfmittel aussehen mögen, verschliesst jeden Zugang zur Wahrheit (s. darüber auch in: "Über historische Treue" in Karl Wolfskehl: Bild und Gesetz).28

Auch zwanzig Jahre nach dem Erscheinen des zitierten, 1927 veröffentlichten Essays "Über die historische Treue" haben die darin formulierten fundamentalen Sätze, die einst im George-Kreis Communis opinio waren, ihre Geltung.29 Die geheime Botschaft des Essays lautete: Mythos statt Geschichte. Die attackierte moderne Geschichtsbetrachtung ("Analogie, und wie dergleichen Hilfsmittel aussehen mögen"), die sich Ende des 19. Jahrhunderts als Historismus etablierte, beinhaltete ja Arbeit am Vergangenen, die zwangsläufig eine Entmystifizierung bedeutete. Im Essay hob Wolfskehl insbesondere "das Recht zur magischen Geschichtsbetrachtung in uns" hervor, das ihm abgesprochen zu werden drohte.30 Der Historismus wurde (nicht nur von den Georgianern) als Relativismus gescholten, denn er würde den Blick auf die charismatischen Persönlichkeiten bzw. Helden der Geschichte, das Geheimnisvolle und Ewige, d. h. das "unbedingte Wirken" von Geschichtlichem, beeinträchtigen. Die Kritik am mangelnden Respekt vor dem Heiligen, dem geschichtlichen Gewordensein und der mythischen Zeit ist eine Kritik am "Opportunismus gegenüber dem Massengeschmack", wie Stefan Breuer in seiner erkenntnisreichen Studie Der ästhetische Fundamentalismus (1995) über die sozialpolitischen Aspekte im George-Kreis argumentierte.31 Insbesondere in der Zeit der Weimarer Republik, in der sich die moderne Zivilisation ausprägte, werden auch von Karl Wolfskehl Schutzzonen der geistigen Reserven etabliert: Das kulturelle Erbe besteht u. a. aus Goethe, Jean Paul, Hölderlin, der Bibel, Mythologien, Legenden und natürlich immer wieder Stefan George, der bereits selbst diese Erbschaft vereinnahmt hatte. Die Dichotomie Kultur - Zivilisation implizierte damals ebenfalls eine rabiate Abwehr der anglo-amerikanischen, slawischen und sogar protestantischen kulturellen Einwirkungen auf Deutschland, die als "entseelung von All und Mensch" und als Entgleisung, des Elitegeistes gedeutet wurde. ${ }^{32}$

\section{BaN I, S. 329 f.}

29 GW II, S. 383-389.

$30 \mathrm{GW}$ II, S. 384. Hervorhebung von Wolfskehl.

31 Breuer, Stefan, Ästhetischer Fundamentalismus. Stefan George und der deutsche Antimodernismus, Darmstadt 1995, S. 1. 
Breuer zitiert mehrere Außerungen Wolfskehls, die auf seinen Kampf gegen die "Barbarei der Moderne" hinweisen. Der Hamburger Soziologe introduziert das vor allem von Martin Riesenbrodt abgeleitete Konzept des Fundamentalismus, welches er als Gegenaufklärung definiert, weil diese ästhetische Opposition gegen die rationalen Tendenzen und deren Institutionalisierung in der westlichen Welt zur als antimodern gedeuteten "Reinterpretation der religiösen Tradition" führte. 33

Wie wir gesehen haben, deuten die stilistischen Mittel in Wolfskehls lyrischen Texten auf ein bewußtes Bremsen von Zeit, auf Mechanismen des Zeitschindens hin. Weiter: Epigonalität wird als Strategie der Konservierung mythischer, biblischer und eigener Schätze eingesetzt. Diese Selbstblendung, die die Wirklichkeit ausblendet, bekämpft nicht nur eine moderne Literaturauffassung, die um 1900 einen bedeutenden Schub bekam und im Zeichen der Authentizität stand, sondern im gleichen Atemzug die in der gesellschaftspolitischen Moderne dominierenden Beschleunigungsprozesse, die sich dem Fortschritt und der Rationalität verschrieben hatten. Die Welt wurde entzaubert.34 In diesem Sinn sollte m. E. Walter Benjamins Erinnerung an den sechzigjährigen Wolfskehl anders, nämlich kritischer gedeutet werden. In seinem Text betont Benjamin jene Schrift bzw. Schreibweise Wolfskehls, "von der eine Graphologin gesagt hat, sie bedürfe 'gradezu eines Schlüssels, um überhaupt gelesen zu werden'. Und sie gleicht ihrem Schreiber darin, daß sie ein unvergleichliches Versteck von Bildern ist."35 Im Zuge seines materialistischen Kunstverständnises ordnete Benjamin Karl Wolfskehl einem unzeitgemäßen Raritätenkabinett zu, denn dieser war einer der letzten Vertreter einer in der "unwirtlichen, blendenden Epoche der großen Industrie" mit ihrem "genormten, denaturalisierten Dasein der zivilisierten Masse" untergehenden auratischen, sakralen Kunst.36

32 Ebd., S. 200.

33 Ebd., S. 2-4 und 238.

34 Vgl. Habermas, Jürgen, Der philosophische Diskurs der Moderne. Zwölf Vorlesungen, Frankfurt a. M. 1985, S. 13. Habermas' Erklärungsansatz der Moderne ist die sich- auf Max Weber, Benjamin Nelson und Reinhard Koselleck stützende - interne Beziehung zwischen Modernität und Rationalität und die Entzauberung der Welt.

35 Benjamin, Walter, "Karl Wolfskehl zum sechzigsten Geburtstag." a. a. O., S. 368 .

36 Benjamin, Walter, “Über einige Motive bei Baudelaire.” In: Ders., Gesammelte Schriften I. 2. Hg. von Rolf Tiedemann und Hermann Schweppenhäuser, Frankfurt a. M. 1974, S. 605-654. 
Jedoch: Die zahllosen Spiegelungen in Wolfskehls Texten bedeuten nicht unbedingt einen Rückschritt, sind nicht nur als literarische Kleinmeisterei abzutun. Die Kategorie der Epigonalität sollte vielleicht durch die der Intertextualität - als Technik der Selbstbegegnung und -findung, nicht sosehr der Selbstverwirklichung - ersetzt werden. 37 Wie dem auch sei, die auch während und nach der Zeitwende 1933 vorangetriebene Vervielfältigungs- und Reproduktionslust von Vor-Geschriebenem hatte zur Folge, daß der Lyriker Opfer seiner Epigonalität bzw. intertextuellen Methode wurde: Als er im Gedicht "Auf Erdballs letztem Inselriff" von Neuseeland aus seine Existenz überblickte, schlug er radikaler als vorher seinen bewährten Ton der Entsagung an, den er bereits um die Jahrhundertwende im dritten Teil von "Blondel" aus den Gesammelte[n] Dichtungen gewählt hatte. Darin heißt es in der zweiten Strophe (wieder in direkter Korrespondenz zu George): "Müde müde glieder beben, / Lied und leid, / Von dem söller schwalben schweben, / Bergen ferne sich in reben, / Leid und lied." In der Wiederbegegnung des Exulanten mit seinem früheren lyrischen alter ego und Gedicht klingt der Vers jetzt endgültiger: "Ob mich auch Frohsinn mied, / Einschläft das Weh, das Leid wird Lied."38 (Z. 5-6) Das Ich auf "Erdball letztem Inselriff" ist nur noch schemenhaft als Traumgestalt vorhanden, da gefiltert durch die eigenen Texte. Die im lyrischen Frühwerk erarbeitete intentionelle "innere Emigration" (Stichwort: Exul Poetica) verfestigt sich schlußendlich in der "äußeren Emigration", d. h. im existentiellen Exil. An den Antipoden wird das Projekt der Antimoderne vollendet.

37 Vgl. Mattenklott, Gert, "Epigonalität." In: Ders., Blindgänger. Physiognomische Essais, Frankfurt a. M. 1986, S. 72-100.

$38 \mathrm{GW} \mathrm{I}, \mathrm{S} .15$ und 225. Hervorhebungen von mir. 